\title{
Linking and comparing short and full-length concept inventories of electricity and magnetism using item response theory
}

\author{
Yang Xiao, ${ }^{1,2}$ Joseph C. Fritchman, ${ }^{2}$ Jacqueline Y. Bao, ${ }^{2}$ Ying Nie, ${ }^{1,3}$ Jing Han, ${ }^{2}$ Jianwen Xiong, ${ }^{1}$ \\ Hua Xiao, ${ }^{1, \dagger}$ and Lei Bao $\circledast^{2, *}$ \\ ${ }^{1}$ South China Normal University, Guangzhou, Guangdong 510006, China \\ ${ }^{2}$ The Ohio State University, Columbus, Ohio 43210, USA \\ ${ }^{3}$ Education Bureau of Zhangjiagang, Zhangjiagang, Jiangsu 215600, China
}

(Received 5 June 2019; published 9 December 2019)

\begin{abstract}
In physics education research (PER), concept inventories (CIs) have become standard instruments for assessing students' learning throughout instruction. To promote widespread use of concept inventories, previous studies have developed an approach to split a full length CI into short versions of CIs. This research extends the existing method to fully utilize the item response theory framework in equating and linking between the short CIs and the full length CIs. Three quantitative studies have been conducted: First, the extended algorithm is applied to divide the Brief Electricity and Magnetism Assessment (BEMA) into two half-length BEMAs (HBEMAs). Through a series of test-equating and validation analysis, the HBEMAs are confirmed to measure the same latent constructs of student understanding of electricity and magnetism to that of the original BEMA at a similar level of reliability. The second study establishes equivalent score conversions among the three versions of BEMA using the Stocking-Lord method, which has the best performance on equating error reduction among several methods explored. It is also confirmed that the equivalent statistical characteristics of the three versions of BEMA are equity and population invariant. In the third study, the extended algorithm is applied to link and compare the BEMA and the Conceptual Survey of Electricity and Magnetism (CSEM). After linking the BEMA and CSEM assessment scales, it becomes possible to directly convert and compare students' performances on the two CIs. It is found that the scales of BEMA and CSEM are almost identical after scale transformation. Based on these studies, it can be suggested that all short and long versions of BEMA and CSEM can be used interchangeably after scale transformation.
\end{abstract}

DOI: 10.1103/PhysRevPhysEducRes.15.020149

\section{INTRODUCTION}

In physics education research (PER), concept inventories (CIs) are commonly used for assessing students' learning throughout instruction [1]. There are also CIs developed to assess the same or similar content. For example, the Conceptual Survey of Electricity and Magnetism (CSEM) and the Brief Electricity and Magnetism Assessment (BEMA) are both designed to assess student understanding of introductory electricity and magnetism [2,3]. Given these resources, parallel and short CIs can be developed, which can

\footnotetext{
*orresponding author. bao.15@osu.edu

Corresponding author. xiaoh@scnu.edu.cn
}

Published by the American Physical Society under the terms of the Creative Commons Attribution 4.0 International license. Further distribution of this work must maintain attribution to the author(s) and the published article's title, journal citation, and DOI. provide valuable utilities in assessment such as saving testing time and avoiding test-retest effects [4].

In this study, the item response theory (IRT) framework was incorporated to extend an existing algorithm for developing short equivalent CIs [5]. The extended algorithm was successfully applied to develop and validate two short equivalent versions of BEMA. In addition, the extended algorithm was used to link and compare students' performances on CSEM and BEMA, which are two different CIs targeting similar content.

\section{A. Developing and equating short version CIs}

In a number of recent studies, Han et al. and Xiao et al. have developed and improved a set of methods for creating and validating equivalent short CIs based on the original full-length CIs [5,6]. In Han et al.'s study, the algorithm for developing equivalent and short parallel CIs involves five steps: (i) classify the items from the original CI into categories based on content analysis; (ii) split the items into two short versions to minimize their differences in both content and difficulty; (iii) compare the assessment features 
of the short CIs and the original CI; (iv) establish linear regression models between the total scores of any two of the CIs; and (v) reexamine the score conversion model using a different sample [6]. Using the algorithm, Han et al. developed two half-length versions of force concept inventory (FCI) and validated the assessment equivalence among the three versions of the FCI (two half-length tests and the original test) $[6,7]$.

Recently, Xiao et al. further extended Han et al.'s algorithm with an emphasis on incorporating standard practices in test equating, which are expected to meet five common requirements including equal construct, equal reliability, symmetry, equity, and population invariance requirements $[5,8-10]$. In the new study, the linear regression model of equating used in Han et al. [6] was replaced with other methods due to the asymmetry between the regression relations obtained from two equatable tests [4,11]. In Xiao et al.'s study, a symmetric equating model was used and a seven-step revised algorithm for developing and validating short CIs was proposed [5]: (i) conduct content analysis on the CI questions to develop splitting categories, (ii) create and adjust the question assignment in short CIs based on existing data and content categories, (iii) examine the equal construct requirement, (iv) examine the equal reliability requirement, (v) establish equating functions among different CIs, (vi) examine the equity and population invariance requirements, and (vii) apply new CIs in randomized testing to validate the CIs and equating model. Typically, the development of short CIs would go through multiple cycles of revisions and reevaluation until all requirements are met. Following the revised algorithm, two short versions of CSEM have been developed and validated [5].

While the revised algorithm by Xiao et al. has addressed some of the limitations identified in previous studies, the equating functions were established solely based on classical test theory (CTT) [5]. In recent researches, testing equating using item response theory has been gaining popularity as it provides a more integrated psychometric framework for analyzing tests designed with different forms and contexts. For example, it is possible to conduct complex equating among multiple test forms connected through different paths [12] and among tests under multidimensional assumption [13]. Therefore, in order to further refine the short-CI development method it is valuable to incorporate the IRT framework in the test equating algorithms, which also makes it practical to directly compare results obtained from different CIs targeting similar content.

\section{B. Comparing different CIs targeting similar content}

In the literature, a few studies have compared different CIs targeting similar content. For example, using basic statistics (e.g., item difficulty, discrimination, correlation, normalized gains, Cronbach's $\alpha$, etc.), Thornton et al. compared the assessment features of the FCI and the force and motion conceptual evaluation (FMCE) [14-16]. They found that while the correlation between the two tests was quite substantial (approximately 0.78), the mean pretest score on the FCI was significantly higher than that on the FMCE, which often leads to different normalized gains for an identical population. Therefore, it was concluded that these two CIs do not measure the same conceptual domains.

Comparisons between CSEM and BEMA have also been conducted. In 2008, Pollock found that while the mean score on the BEMA was significantly higher than that on the CSEM, the normalized gains of these two CIs were statistically indistinguishable [17]. It was suggested that the CSEM and BEMA were nearly equivalent in measuring students' understanding of static electricity and magnetism at the introductory level. Recently, Eaton et al. corroborated Pollock's findings [18]. The two CIs were compared using both the CTT and IRT approaches. It was found that the overall difficulties of the two CIs were essentially identical. Between the two CIs, there are 7 similar items. The CTT item analysis suggested minor differences on these 7 items regarding their item difficulty, discrimination, and pointbiserial correlation. Despite the differences, the 7 items were used as common items for linking the measurement scale of each CI for comparing their IRT results. After test linking procedures, it was found that CSEM had slightly better test information than BEMA for the typical range of student abilities.

Ideally, the common items used in IRT equating to link different tests should be identical and span a range of content areas representative of the full test [4]. The 7 linking questions in Eaton et al.'s study have substantial differences in number of choices and statistical characteristics. In addition, the content of the 7 questions only span a narrow range of that of the entire test (see Figs. 9 and 10 in the Appendix). Therefore, using these 7 questions as common linking questions could generate nontrivial uncertainties and can raise concerns in the validity of the results. In this study, identical common items will be used in order to remedy this issue and provide additional evidence on the validity of the results from previous work.

Furthermore, although the overall difficulty of CSEM and BEMA has been compared as being equivalent in previous studies $[17,18]$, there is a gap in the literature on finer grained comparisons of individual students' performances on these two CIs which is worth exploring. In addition, this study will produce two short CIs of BEMA, which make a total collection of 6 CIs including short and long versions on electricity and magnetism. Therefore, conducting a detailed comparison among all these versions as well as developing validated equating models for these CIs will contribute to the literature and provide valuable resources for researchers and educators in assessment of electricity and magnetism. From the methodology perspective, the method developed in this study can provide a general approach for making direct 
comparisons of students' learning outcomes measured with different CIs targeting the same content. Therefore, results of this study will contribute to the literature on methods for equating and comparing different CIs that can be extended to other content areas.

\section{Analyzing CIs using item response theory}

The item response theory (IRT) often references to a family of logistic modeling methods including the popular Rasch model [19,20], which together provide an integrated psychometric framework for modeling students' latent abilities based on test scores. IRT has been widely used in analyzing CIs in PER. Many different IRT models have been used to analyze the psychometric characteristics of CIs, which included the three-parameter logistic (3PL) model, the two-parameter logistic (2PL) model, the Rasch model under unidimensional assumption, and the multidimensional IRT models (see more details in Sec. II. C).

In one of the early studies that applied IRT to analyze CIs, [21] the 3PL IRT model was used to analyze the FCI pretest data collected from 2802 students taking intro level mechanics courses. The results supported the assumption of unidimensionality for IRT analysis of FCI data and the overall fit of each item. Meanwhile, nearly all FCI items showed reasonable item parameters. In recent research and development, the use of the IRT family of analysis methods has gained popularity in PER, especially in the area of assessment development and validation [22-25]. For example, following the Rasch modeling procedures of assessment development [20], Mešic et al. applied the Rasch model to develop the wave optics item bank [22]. The IRT modeling approach has also been applied to analyze twotier instruments [24,25]. Scotti di Uccio et al. developed and validated a two-tier format quantum mechanics evaluation (QME) using both CTT and Rasch analysis [23].

For test evaluation with IRT, Ding reevaluated the unidimensionality assumption and differential item functioning (DIF) of BEMA using Rasch model [26]. The results of Rasch analysis suggested that although BEMA covered a broad range of topics in static electricity and magnetism, the entire test provides a good measure of an overall unidimensional construct of concepts in electricity and magnetism. Regarding differential item functioning, which shows the extent to which the item might be measuring different abilities, five items of BEMA were identified to show differential functioning. In another study conducted by Traxler et al., the DIF analysis was used as a way to evaluate item fairness in the FCI using the 2PL IRT model [27], which detected 11 questions with large DIF. These questions were considered as having strong bias and were recommended to be removed in certain testing circumstances.

Several recent studies also applied multidimensional IRT to evaluate the construct dimensions of a CI through exploratory and or confirmatory approaches [28-30]. Scott and Schumayer [30] used the multidimensional IRT model to explore the factor construct of a FCI dataset [31], which comfirmed an optimal five-factor structure for FCI. Taking the comfirmatory approach using multidimensional IRT modeling, Stewart et al. [28] tested a set of theoretical models of the FCI constructed from expert solutions using 4716 post-test student response. The results suggest that the FCI can differentate student's understanding of Newton's 1 st and 2nd law, of one-dimensional and three-dimensional kinematics, and of the principle of the addition of forces from Newton's 2nd law.

In the current literature, IRT has been mostly applied to evaluate and validate test items, which has generated valuable understanding of the assessment features of many popular CIs. In this study, a new research focus will be explored, which applies IRT to develop equating or linking models among different CIs that target similar content, namely, the 6 short and long versions of CSEM and BEMA. The results of this work can provide utility tools and methodology practices for comparing assessment outcomes of different CIs targeting similar content. It should be noted that while both test equating and linking use similar statistical processes to connect scores on different tests so that the results can be directly compared, their definitions can be further clarified [4]. Following the tradition, "equate or equating" is used to describe the relationship between scores on different versions of the same test such as between HBEMAs and BEMA. Meanwhile, "link or linking" is used to describe the relationship between scores of different tests such as between BEMA and CSEM.

\section{Research questions}

Based on the review of current literature, three areas of research are found valuable to further pursue. First, it is valuable to develop short versions of BEMA, which should aid the utility of applying the BEMA family of assessment in practice. Second, since there are a collection of 6 short and long versions of assessment on static electricity and magnetism, it would useful to develop equating and linking models among these versions so that scores from one test can be compared with another. Finally, it is also valuable to fully evaluate and compare the assessment features of BEMA and CSEM, which are linked with identical common items. The results will be compared with the literature and contribute to develop a more solid knowledge base for researchers and educators on how to properly use the two CIs in practice and interpret their results.

Specifically, this study seeks to answer the following three research questions:

RQ1: To what extent can the half-length versions of BEMA (HBEMAs) be equated to the BEMA?

RQ2: To what extent can the BEMA be linked to the CSEM?

RQ3: To what extent do the BEMA and the CSEM differ in assessing students' understanding of electricity and magnetism? 
To answer the first research question, the full-length BEMA test will be divided into two short CIs following the method in Xiao et al. [5], but with an extension into using the IRT framework in addition to the CTT approaches for test evaluation and validation. To address the issue of nonidentical common items in Eaton et al.'s study [18], the study will use identical items in HBEMAs and HCSEMs as common items for linking between the two full-length tests. Using identical common linking items, more stable linking results between the full-length BEMA and CESM can be obtained and used to develop a score conversion model between the two commonly used CIs. The results will answer the second research question and will also be compared with the outcomes from Eaton et al. [18]. To answer the third research question, the linked results of BEMA and CSEM are compared to identify the similarities and differences of the two CIs on assessing students' understanding of electricity and magnetism.

\section{METHODOLOGY}

\section{A. Data and instrument}

To evaluate the equivalence of the two HBEMAs and the original BEMA, two datasets (referred as DS1 and DS2) were collected from two different college populations using the full BEMA $\left(N_{\mathrm{DS} 1}=330, N_{\mathrm{DS} 2}=619\right)$. The DS1 was collected in a large state university with a U.S. ranking of top 60 and an acceptance rate of 52\%. The DS2 was collected at another large state university with a U.S. ranking of top 100 and an acceptance rate of $60 \%$. To link between BEMA and CSEM, DS1, DS2, and two additional datasets (referred as DS3 and DS4) were used. DS3 was collected with the full CSEM at another large state university $(N=1422)$ with a U.S. ranking of top 130 and an acceptance rate of 70\%. DS4 was collected with the full CSEM from a Chinese high school $(N=690)$, which is a regional top-tier school with a college admission rate of $90 \%$.

For the university populations, students were enrolled in a calculus-based electromagnetism course. These students took the BEMA or CSEM as a pretest during the first week of the course and as a post-test during the week before final exam. Only the post-test scores were used for analysis because the students were mostly guessing on the pretest as suggested by their scores $(20 \%-30 \%)$. For the post-test, students' mean scores on BEMA and CSEM were around 50\% (see Table II).

In the Chinese high school, students were randomly assigned to take one of two versions of mixed tests, each of which included one of the HBEMAs developed in this study and one of the HCSEMs developed in the previous study [5]. The two versions of mixed tests were constructed as version 1 (HBEMA1-HCSEM1 in random order) and version 2 (HBEMA2-HCSEM2 in random order). As a result, each mixed test is composed of 33 items with 16 from BEMA and 17 from CSEM. These tests are translated Chinese versions that have been validated and applied in previous studies $[5,32]$.

The Chinese high school students only took the mixed tests as a post-test after they completed the learning of all the content related to electricity and magnetism in grade 10 . To counter the possible impact from testing orders, the two halves of a mixed test were given to each student in random orders. The results show that students' average scores on the mixed tests are comparable to that of the U.S. college students (around 50\%, see Tables II and IX), and therefore, all populations are considered to be similar in performance level.

\section{B. Developing and validating the half-length versions of BEMA}

In this study, two half-length versions of BEMA were developed and validated using the first 6 steps proposed in Xiao et al. [5], which will be only briefly discussed here.

In step 1, after conducting a content analysis on the items in BEMA by a team of experts in physics education research, the items were classified into 12 content clusters (see Content cluster in Table I). In step 2, the items were split into two short CIs, cluster by cluster, to minimize the mean difference in the results of the questions on each test (see HBEMA1 and HBEMA2 in Table I). The Rasch item difficulties were used to guide the difference minimization process [26]. Finally, it was found that the average item difficulties for BEMA, HBEMA1, and HBEMA2 were $0.001,-0.001$, and -0.002 , respectively (see Table II).

In the following four steps, five common requirements for test equating were evaluated. In step 3, the requirement

TABLE I. The content clusters assessed in the two short versions of BEMA. The numbers listed under each test reflect item numbers on the original BEMA. Common items used in both half-length tests are marked with an asterisk (*).

\begin{tabular}{lcc}
\hline \hline Content cluster & HBEMA1 & HBEMA2 \\
\hline $\begin{array}{l}\text { Coulomb's law } \\
\text { Superposition principle of }\end{array} \quad 3$ & 2 \\
$\quad$ Electric field & 5 & 4 \\
$\begin{array}{l}\text { Interactions between electric } \\
\quad \text { charges and fields }\end{array}$ & $6^{*}$ & $6^{*}$ \\
$\begin{array}{l}\text { Potential difference in electric } \\
\quad \text { field }\end{array}$ & $15,16^{*}$ & $14,16^{*}$ \\
$\begin{array}{l}\text { Gauss's law } \\
\text { Insulator and conductor }\end{array}$ & 18 & 18 \\
$\begin{array}{l}\text { Mobile charges in conductor } \\
\text { Circuits }\end{array}$ & 7 & 19 \\
Lorentz force & 8 & 9 \\
Magnetic field & $13^{*}, 17^{*}$ & $12,13^{*}, 17^{*}$ \\
Biot-Savart law and magnetic field & $22^{*}$ & 25,26 \\
$\quad$ of a loop & $24^{*}$ & $24^{*}$ \\
Faraday's law & 30 & 29 \\
\hline \hline
\end{tabular}


TABLE II. Mean scores comparisons of the two HBEMAs and the full-length BEMA. The first row of data gives the mean values and standard deviations of average item difficulty based on the Rasch analysis outcomes of BEMA [26]. For DS1 and DS2, the mean and standard devotion (SD) of students' scores are computed in percentage scale.

\begin{tabular}{lccc}
\hline \hline & & \multicolumn{2}{c}{ Mean $(\mathrm{SD})$} \\
\cline { 2 - 4 } Dataset & BEMA & HBEMA1 & HBEMA2 \\
\hline Rasch item difficulty & $0.001(0.921)$ & $-0.001(0.754)$ & $-0.002(0.749)$ \\
DS1 (mean score) & $48.44(18.45)$ & $49.26(21.12)$ & $47.94(20.69)$ \\
DS2 (mean score) & $52.75(17.23)$ & $54.14(19.06)$ & $51.88(18.69)$ \\
\hline \hline
\end{tabular}

on equal construct was evaluated using both unidimensionality tests and correlation analysis. The process and results of unidimensionality tests are included in the Supplemental Material [33]. Under the unidimensional assumption, which allows for the application of unidimensional IRT to analyze the three CIs, the correlations of students' estimated abilities between any two of the CIs were calculated to examine their construct similarity.

In step 4 , the equal reliability requirement was examined using the reliability coefficients estimated by $2 \mathrm{PL}$ IRT models for the three CIs. Because the test lengths were different, the Spearman-Brown prophecy formula was used to adjust the reliabilities of the two HBEMAs to the equivalent values of a 30 -item test, which is the length of the full BEMA.

In step 5, the symmetry requirement is automatically satisfied after establishing the equating function. To equate among the three CIs, linear transformations between score scales of any two CIs need be determined. To do this, common items and four different equating methods were used (see more details in the next section). The performance of the four equating methods were compared and the best performing method was used to conduct final test equating.

In step 6, the equity and population invariance requirements were further examined. The equity requirement of the three CIs can be examined using test characteristic curves (TCCs) of different CIs. The TCC, which is the sum of the all the item response functions $P_{j}\left(\theta_{i}\right)$ at a given ability $\theta_{i}$, can visually show a functional relation between students' model predicted scores and their estimated abilities [34]. In this study, the 2PL model was used as the item response function (see more details in the next section). For direct comparison between the three versions of BEMAs, the TCCs were all transferred to the percentage scale, given as

$$
S_{i}=\frac{1}{J} \sum_{j=1}^{J} P_{j}\left(\theta_{i}\right) .
$$

Here, $S_{i}$ is the model predicted score for examinees having ability $\theta_{i}$ on a test with a total of $J$ items. When the three CIs share parallel and similar TCCs, they can be considered as equivalent instruments, producing equivalent model scores for a given ability.
The equity requirement of the three CIs can also be examined by comparing students' performances on different CIs. The equity requirement is satisfied when students achieve equivalent measured or equated scores on the three CIs. To compare students' scores on two tests, the rootmean-square deviation (RMSD) was used [6] given as

$$
\operatorname{RMSD}_{X Y}=\sqrt{\frac{\sum_{i}^{N}\left[S_{i}(Y)-S_{i}(X)\right]^{2}}{N}}
$$

Here, $S_{i}(X)$ and $S_{i}(Y)$ are the measured or equated scores of the $i$ th student with two different tests labeled as $X$ and $Y . N$ is the number of students.

The population invariance requirement can be evaluated from the standardized mean differences (SMD) between the performances of different subpopulations on different tests $[8,35]$. For example, the SMD between scores of two subpopulations DS1 and DS2 on the three tests are computed using

$$
\mathrm{SMD}_{X}=\frac{\mu_{\mathrm{XDS} 1}-\mu_{\mathrm{XDS} 2}}{\mathrm{SD}_{\mathrm{Xtotal}}},
$$

where $\mu_{\mathrm{XDS} 1}$ and $\mu_{\mathrm{XDS} 2}$ are the mean scores on test $X$ from DS1 and DS2, respectively, and $\mathrm{SD}_{\mathrm{Xtotal}}$ is the standard deviation of test $X$ from the two subpopulations combined. The SMD provides a measure of the variation between mean scores of subpopulations as a fraction out of the total population's standard deviation, which can be used to evaluate the dependence of the equating function on subpopulations [11]. Since the IRT framework was emphasized in this study, the values of SMD were also computed using students' estimated ability.

\section{Linking and equating CIs using item response theory}

Item response theory models are commonly used to link or equate results of different tests [4]. One of the most widely used is the 3PL model, which assumes that each item $j$ has three parameters (i.e., the discrimination $a_{j}$, difficulty $b_{j}$, and the pseudochance level $c_{j}$ ). The 3PL model can be simplified into a 2PL model, which sets the pseudo-chance level parameter to be zero $\left(c_{j}=0\right)$, and the Rasch model, which further sets the discrimination parameter equal to 1 for all items $\left(a_{j}=1\right)$. For this study, the 2PL 
model was used because the 3PL model has been questioned when analyzing distractor driven CIs [36]. In the 2PL model, the probability that person $i$ with a given ability $\theta_{i}$ will correctly answer item $j$ is given by

$$
P_{i j}\left(x=1 \mid \theta_{i}\right)=\frac{\exp \left[a_{j}\left(\theta_{i}-b_{j}\right)\right]}{1+\exp \left[a_{j}\left(\theta_{i}-b_{j}\right)\right]} .
$$

In this study, parameters of the 2PL model on different tests were estimated separately under the common item design, in which each pair of tests are designed to include a number of common items and the two tests are each given to one of two examinee groups. In this case, the common items will produce two sets of estimated parameters. Then, a linear transformation with the slope $A$ and intercept $B$ is used to convert the two sets of parameters into a single scale [4]. Using the transformation coefficients (slope $A$ and intercept $B$ ), parameters of the remaining items of the two equating tests $\left(X\right.$ and $Y$, ) can be converted: $a_{X i}=a_{Y i} / A$, and $b_{X i}=A b_{Y i}+B$, where $a_{Y i}$ and $b_{Y i}$ are parameter estimates for item $i$ in test $(Y)$, and $a_{X i}$ and $b_{X i}$ are the corresponding parameters of test $(X)$.

To estimate the transformation coefficients, four methods are commonly used (e.g., the mean-mean, mean-sigma, Haebara, and Stocking-Lord methods) [4]. The first two methods use the mean $(\mu)$ and standard deviations $(\sigma)$ of parameters for the common items. Using the mean-mean method, the transformation coefficients are given as $A=$ $\mu\left(a_{Y}\right) / \mu\left(a_{X}\right)$ and $B=\mu\left(b_{X}\right)-A \mu\left(b_{Y}\right)$, where the subscripts $Y$ and $X$ indicate the parameters $a$ or $b$ estimated using data of the test $Y$ and test $X$, respectively. Using the mean-sigma method, the transformation coefficients are given as $A=\sigma\left(b_{J}\right) / \mu\left(b_{L}\right)$ and $B=\mu\left(b_{J}\right)-A \mu\left(b_{L}\right)$. While the previous two methods only consider partial item parameter estimates for common items simultaneously, the Haebara and Stocking-Lord methods take all of the item parameters into account simultaneously [4]. Specifically, the Haebara approach computes the transformation coefficients that minimize the sum of the squared difference between the item characteristic curves (IRCs) for common items in two test forms, and the Stocking-Lord method uses TCCs in the minimization process (see more details in Ref. [4]). It has been demonstrated that the Haebara and Stocking-Lord methods always produce more accurate equating results than the previous two methods.

When the best solution of the transformation coefficients is determined, the next step is to find the relationships between measured scores on a pair of tests. Students' scores on CIs are usually based on their numbers of correct responses. In this study, the IRT observed-score equating method is used to establish the score conversions. To do this, the conditional observed-score distribution $\left[f\left(x \mid \theta_{i}\right)\right]$, which gives the probability distribution of observing a score of $x$ at a given ability $\theta_{i}$. This distribution is calculated from a compound binomial distribution based on item parameter estimates using the recursive formula proposed by Lord and Wingersky [37]. Then, these observed-score distributions are accumulated over a population of examinees with different abilities to produce a number-correct observed-score distribution for a specific test $[f(x)]$. For a population of examinees with continuous ability distribution $\psi(\theta)$, the number-correct observed score distribution is given by $f(x)=\int f(x \mid \theta) \psi(\theta) d \theta$. When the numbercorrect observed score distributions have been obtained following the above procedures, conventional equipercentile equating methods are used to find equating relationships between number-correct scores for a pair of tests (see more details in Ref. [4]).

The above procedures are used to equate three versions of BEMA and to link the original BEMA to CSEM. To equate the three versions of BEMA, each pair of tests are directly equated using their common items. To link the BEMA and CSEM, two mixed tests were used. Specifically, the first mixed test was composed of the HBEMA1 and HCESM1 (labeled as HE\&M1), and the second mixed test was composed of the HBEMA2 and HCEMS2 (labeled as HE\&M2). Each of the mixed tests contains 33 items with 16 from BEMA and 17 from CSEM. Finally, the original BEMA and CSEM are linked through two different paths (see Fig. 1) with one going through HE\&M1 and the other going through HE\&M2, producing two sets of equating coefficients. These equating coefficients are weighted or averaged using the bisector method proposed by Battauz [12].

\section{Comparing the scales of and students' performance on the BEMA and CSEM}

In the IRT framework, the uncertainty in estimating latent ability [i.e., standard error (SE)] can be determined for each possible value along the entire ability scale. Taking the reciprocal of this uncertainty, the item information function (IIF) and test information function (TIF) can be obtained. In this case, the information can be interpreted as how well students' latent ability can be estimated by an item or test as a function of latent ability. For the 2PL model, the item information is given as [38]

$$
I_{j}(\theta)=a_{j}^{2} P_{j}(\theta)\left[1-P_{j}(\theta)\right],
$$

where $a_{j}$ is the discrimination parameter for item $j$ and $P_{j}(\theta)$ is the item response function for item $j$ given by Eq. (4). Considering the local independence held by IRT models, the test information at a given ability level can simply be the sum of the item information at that level.

In this study, the TIFs of the BEMA and the CSEM are used to assess how well the BEMA and CSEM estimate students' understanding of electricity and magnetism. While the information function can be obtained for both item level and test level, the TIF is more useful because the amount of information yielded by a single item is rather small and an examinee's ability is typically estimated with the whole test, not a single item [38]. Specifically, a TIF 


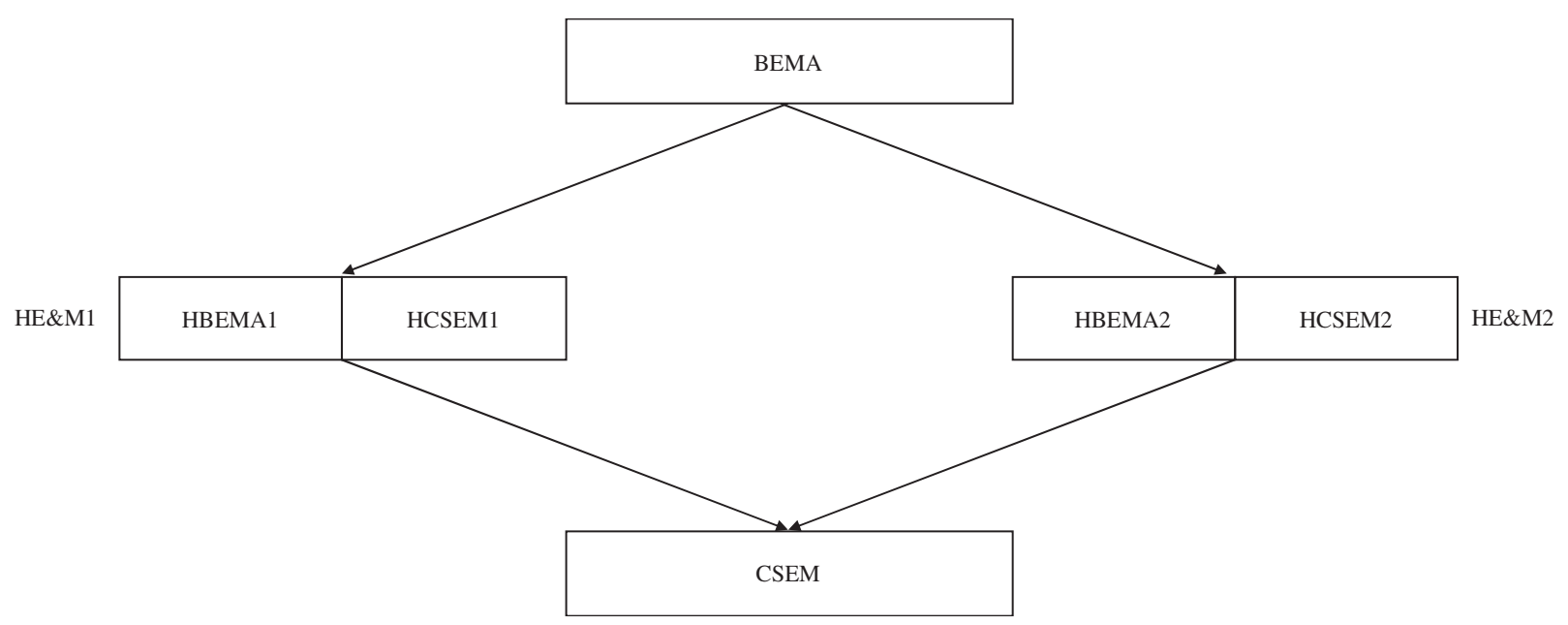

FIG. 1. Linking plan for linking the BEMA and CSEM.

demonstrates how well a test estimates ability over the whole range of ability scores.

Finally, students' performances on the BEMA and CSEM are also compared. The results of DS4, which was collected from 690 Chinese students using the mixed tests of HBEMA and HCSEM, were used to make comparisons between BEMA and CSEM. For this comparison, the measured scores on the half-length CIs were equated back to the full-length CIs using the score conversion methods developed in this and previous studies [5]. Then, a second conversion is conducted to convert the estimated scores on the CSEM to the BEMA scale using the score conversion relationships developed in this study.

With all scores converted into the same scale, students' performances on BEMA and CSEM were compared using paired sample $t$ test, correlation analysis, and equivalence test method. The equivalence test method has been used in the previous study to compare students' performances in different versions of CSEM [5]. The equivalence test follows the two one-sided tests (TOST) procedure proposed by Lakens [39]. In TOST, an upper $\left(\Delta_{U}\right)$ and lower $\left(\Delta_{L}\right)$ equivalence bound should be specified beforehand. When the observed difference falls within the equivalence bounds (i.e., $\Delta_{L}<\Delta<\Delta_{U}$ ), students' performance on the BEMA and CSEM can be considered to be identical [40].

Because students took the a HBEMA and a HCSEM sequentially, the equivalence tests for two dependent means should be used [39], which are given as

$$
t_{L}=\frac{\bar{M}_{1}-\bar{M}_{2}-\Delta_{L}}{\frac{\sqrt{\mathrm{SD}_{1}^{2}+\mathrm{SD}_{2}^{2}-2 \times r \times \mathrm{SD}_{1} \times \mathrm{SD}_{2}}}{\sqrt{N}}}
$$

and

$$
t_{U}=\frac{\bar{M}_{1}-\bar{M}_{2}-\Delta_{U}}{\frac{\sqrt{\mathrm{SD}_{1}^{2}+\mathrm{SD}_{2}^{2}-2 \times r \times \mathrm{SD}_{1} \times \mathrm{SD}_{2}}}{\sqrt{N}}} .
$$

Here, $t_{L}$ and $t_{U}$ are the $t$ values for TOST, $\bar{M}_{1}$ and $\bar{M}_{2}$ are the means of students' scores on each test, $N$ is the sample size for the population $(N=690)$, and $\mathrm{SD}_{1}$ and $\mathrm{SD}_{2}$ are the standard deviations (SD) of students' scores on the two tests. The degree of freedom (d.o.f.) for the $t$ test is similar to the conventional paired sample $t$ test (d.o.f. $=N-1$ ).

To conduct equivalence test, the first step is to determine the equivalence bounds based on the desired power (type II error), $\alpha$ level (type I error), and sample size. With a sample size of 690 , this study set the statistical power at $\beta=80 \%$ and equivalence at the $\alpha=0.05$. These conditions give the equivalence bounds of Cohen's $d_{z}$ being from -0.11 to 0.11 , which corresponds to score boundaries from $-1.91 \%$ to $1.91 \%$. That is, if the difference between students' mean scores on BEMA and CSEM is within $\pm 1.91 \%$, students' performances on the two CIs can be considered as being identical. The equivalence test analysis was conducted using the TOSTER package in $\mathrm{R}$ [39].

\section{RESULTS OF COMPARING THE EQUIVALENCE AMONG THE THREE VERSIONS OF BEMA}

\section{A. Descriptive statistics}

Following the procedures proposed in the previous studies [5,6] and guided with the Rasch analysis outcomes of BEMA [26], two candidate versions of the HBEMAs were designed (see Table I). As a first check of assessment equivalence, the students' mean scores on the full BEMA and the two HBEMAs were evaluated with three different datasets shown in Table II.

As shown in Table II, using the mean values of average item difficulty of all three tests are virtually identical. DS1 and DS2 were from two different populations in a large Midwestern public University. DS1 was collected from students who enrolled in an introductory electricity and magnetism class during spring semester (off-sequence class), while DS2 was collected from students who enrolled 


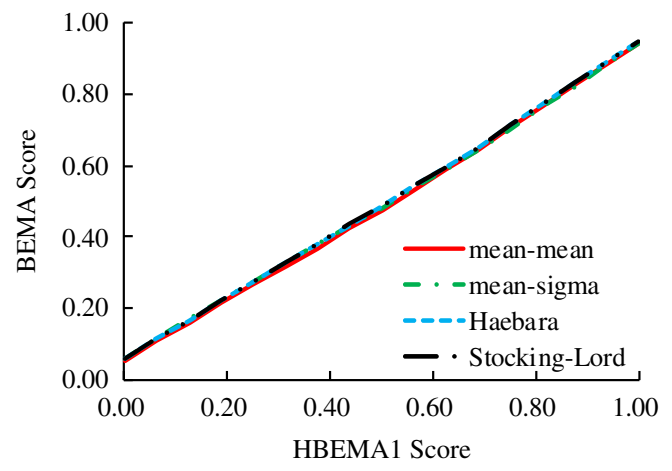

(a)

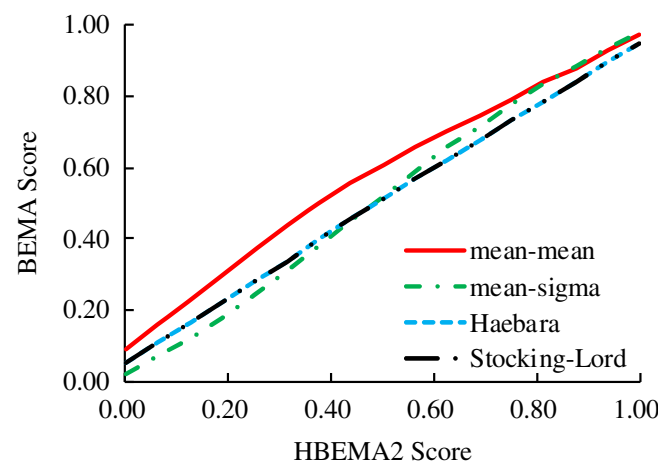

(c)

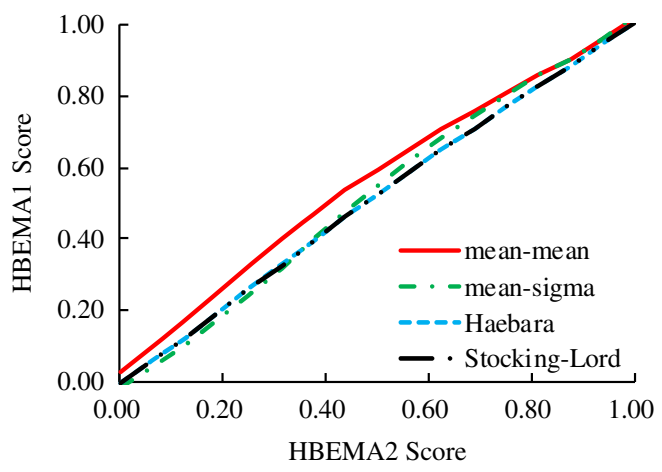

(e)

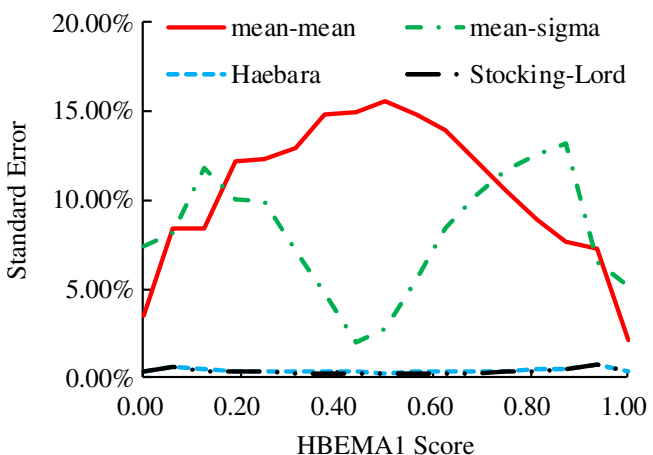

(b)

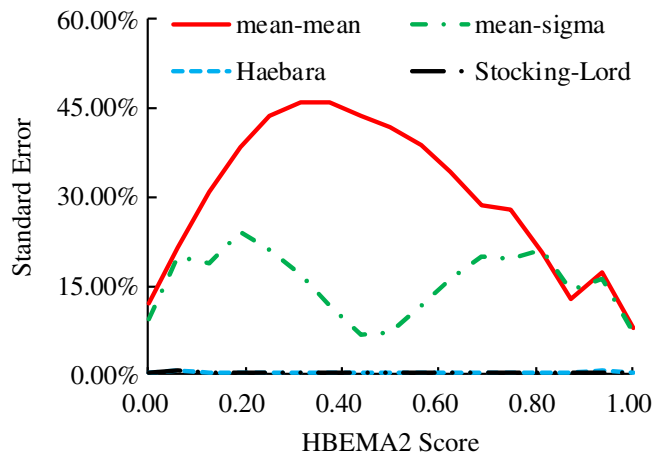

(d)

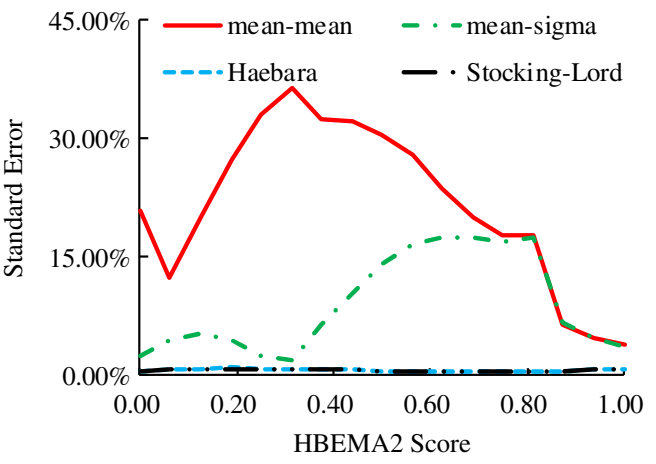

(f)

FIG. 2. Results for equating. (a)-(b) Equating function and SE for equating BEMA and HBEMA1; (b)-(c) Equating function and SE for equating BEMA and HBEMA2; (e)-(f) Equating function and SE for equating HBEMA1 and HBEMA2.

in the same electricity and magnetism class during fall semester (in-sequence class). Apparently, students in the fall semester scored slightly higher than those from the spring semester $(p<0.01$, Cohen's $d$ ranged from 0.203 to 0.246). Within a single dataset, the difference of mean scores between any two of the three tests is less than $2.5 \%$, which is comparable to the results of half-length versions of the CSEM and FCI $[5,6]$.

\section{B. Construct equivalence}

The unidimensionality assumption of the 2PL model requires that BEMA and two HBEMAs measure the same unidimensional latent construct, which can be interpreted as student understanding of introductory electricity and magnetism. To test the unidimensionality of BEMA and its two shorter versions, three methods were used including scree plots, goodness of fit, and the three dimensionalitytest indices (DETECT, ASSI, and Ratio). Together, the results of the unidimensionality analysis suggest that to a large extent BEMA and HBEMAs satisfy the assumption of unidimensionality (see more details in the Supplemental Material [33]).

To assess the extent to which the unidimensional constructs measured by the three CIs represent the same construct, correlation and follow up reduction in uncertainty 
TABLE III. Summary of equating three versions of BEMA using four equating methods.

\begin{tabular}{|c|c|c|c|c|c|c|}
\hline & \multirow[b]{3}{*}{ Methods } & \multicolumn{4}{|c|}{ Equating coefficients } & \multirow{3}{*}{$\begin{array}{c}\text { Mean conditional SE } \\
\text { of measurement } \\
\text { for total score }\end{array}$} \\
\hline & & \multicolumn{2}{|c|}{ A } & \multicolumn{2}{|c|}{$B$} & \\
\hline & & Constant & SE & Constant & SE & \\
\hline \multirow[t]{4}{*}{ BEMA-HBEMA1 ${ }^{\text {a }}$} & mean-mean & 1.01 & 0.05 & 0.06 & 0.85 & $10.63 \%$ \\
\hline & mean-sigma & 1.05 & 0.85 & 0.04 & 0.29 & $8.08 \%$ \\
\hline & Haebara & 1.01 & 0.05 & 0.01 & 0.05 & $0.43 \%$ \\
\hline & Stocking-Lord & 1.02 & 0.05 & 0.01 & 0.05 & $0.37 \%$ \\
\hline \multirow[t]{4}{*}{ BEMA-HBEMA2 $2^{\mathrm{b}}$} & mean-mean & 1.00 & 0.05 & -0.55 & 2.60 & $10.63 \%$ \\
\hline & mean-sigma & 0.74 & 1.01 & -0.06 & 0.33 & $8.08 \%$ \\
\hline & Haebara & 1.00 & 0.05 & 0.01 & 0.05 & $0.43 \%$ \\
\hline & Stocking-Lord & 1.00 & 0.05 & 0.01 & 0.05 & $0.37 \%$ \\
\hline \multirow[t]{4}{*}{ HBEMA1-HBEMA2 $2^{\mathrm{c}}$} & mean-mean & 1.00 & 0.07 & -0.36 & 1.70 & $21.64 \%$ \\
\hline & mean-sigma & 0.81 & 0.66 & -0.10 & 0.59 & $9.09 \%$ \\
\hline & Haebara & 0.99 & 0.06 & 0.00 & 0.06 & $0.78 \%$ \\
\hline & Stocking-Lord & 0.99 & 0.07 & 0.00 & 0.06 & $0.76 \%$ \\
\hline
\end{tabular}

${ }^{\mathrm{a}}$ Item parameters of BEMA are converted to the scale of HBEMA1.
bItem parameters of BEMA are converted to the scale of HBEMA2.
${ }^{\mathrm{c}}$ Item parameters of HBEMA1 are converted to the scale of HBEMA2.

(RiU) indices were computed using students' estimated abilities from two tests [8]. The correlation analysis shows that students' estimated abilities from the two HBEMAs are each significantly correlated with that of the full BEMA: $r=0.949(p<0.001)$ for HBEMA1 and $r=$ $0.950(p<0.001)$ for HBEMA2. The correlation between HBEMA1 and HBEMA2 is also significant, $r=0.880$ $(p<0.001)$. Then, the correlations were further used to compute RiU, which is an index to assess the strength of the relationship between two tests [8]. According to Dorans [8], an $\mathrm{RiU}$ of 0.50 is set as the cutoff value for equating highstakes tests. The correlation between either HBEMA1 or HBEMA2 and the full BEMA corresponds to an RiU over 0.68. It is encouraging that the RiU between HBEMA1 and HBEMA2 (which is 0.53) also exceeds the minimum value of RiU. The results suggest that both HBEMAs and the full BEMA measure the same or very similar constructs, and that students' performances on these three tests can be equated without concerning construct changes.

In summary, the analysis shows that the three CIs all measure a single and similar unidimensional construct. Synthesizing the analysis outcomes, it can be established that the three CIs have achieved construct equivalence.

\section{Reliability equivalence}

To establish evidence for the equal reliability requirement, the expected a posteriori (EAP) reliabilities of the three CIs were used. As a reliability index specified for IRT models, the EAP reliability is comparable to the reliability index Cronbach's $\alpha$ popularly used in the CTT framework. It was found that the values of EAP reliability for the two half-length versions of BEMA are essentially identical (EAP reliability $=0.75, n$ of items $=16$ ). With the increasing test length, the full BEMA achieves a higher value of EAP reliability than that of the HBEMAs (EAP reliability $=0.84$, $n$ of items $=30$ ).

In general, the reliability of a test is affected by both test design and test length. As the test length increases, so does the reliability of the test. Since this study primarily focuses on the effect of test design (i.e., test content changes caused by the HBEMAs) rather than test length, to rule out the effect of test length, the Spearman-Brown prophecy formula is used to calculate the expected reliabilities of both HBEMAs as 30-item tests in keeping with the length of the full BEMA. After the correction, the estimated EAP reliabilities of the HBEMAs achieve an almost identical level to the full BEMA (0.85 vs 0.84 ). The results suggest that the full BEMA and the HBEMAs satisfy the equal reliability requirement after controlling for test length.

\section{Equating HBEMAs and BEMA}

The results for equating HBEMAs to BEMA and for equating HBEM2 to HBEMA1 are plotted as a function of total scores in Fig. 2. As shown in Fig. 2(a), for equating HBEMA1 to BEMA, the four equating methods show very similar trends throughout the range of the total test score. However, for equating HBEMA2 to BEMA and to HBEMA1, there is an obvious difference in performance for the mean-mean method compared to the other three methods in the middle range of the total test scores [see Figs. 2(c) and 2(d)].

The results of the four equating methods were compared based on the standard errors (SEs) of equating, which are 
TABLE IV. Score conversion using the Stocking-Lord method.

\begin{tabular}{|c|c|c|c|c|c|}
\hline \multicolumn{2}{|c|}{ HBEMA1 to BEMA } & \multicolumn{2}{|c|}{ HBEMA2 to BEMA } & \multicolumn{2}{|c|}{ HBEMA2 to HBEMA1 } \\
\hline $\begin{array}{l}\text { Score of } \\
\text { HBEMA1 }\end{array}$ & $\begin{array}{c}\text { Score on } \\
\text { BEMA (SE) }\end{array}$ & $\begin{array}{c}\text { Score of } \\
\text { HBEMA2 }\end{array}$ & $\begin{array}{c}\text { Score on } \\
\text { BEMA (SE) }\end{array}$ & $\begin{array}{c}\text { Score of } \\
\text { HBEMA2 }\end{array}$ & $\begin{array}{c}\text { Score on } \\
\text { HBEMA1 (SE) }\end{array}$ \\
\hline 0 & $1.74(0.11)$ & 0 & $1.62(0.09)$ & 0 & $0.00(0.10)^{\mathrm{a}}$ \\
\hline 1 & $3.46(0.18)$ & 1 & $3.28(0.18)$ & 1 & $0.92(0.13)$ \\
\hline 2 & $5.02(0.13)$ & 2 & $4.95(0.14)$ & 2 & $1.97(0.15)$ \\
\hline 3 & $6.64(0.11)$ & 3 & $6.68(0.12)$ & 3 & $3.04(0.15)$ \\
\hline 4 & $8.24(0.11)$ & 4 & $8.43(0.12)$ & 4 & $4.12(0.15)$ \\
\hline 5 & $9.83(0.09)$ & 5 & $10.17(0.10)$ & 5 & $5.22(0.14)$ \\
\hline 6 & $11.43(0.09)$ & 6 & 11.91(0.09) & 6 & $6.31(0.13)$ \\
\hline 7 & $13.02(0.08)$ & 7 & $13.63(0.08)$ & 7 & $7.38(0.11)$ \\
\hline 8 & $14.63(0.08)$ & 8 & $15.32(0.08)$ & 8 & $8.43(0.10)$ \\
\hline 9 & $16.26(0.08)$ & 9 & $16.99(0.08)$ & 9 & $9.44(0.10)$ \\
\hline 10 & $17.92(0.08)$ & 10 & $18.64(0.08)$ & 10 & $10.42(0.11)$ \\
\hline 11 & $19.61(0.09)$ & 11 & $20.27(0.09)$ & 11 & $11.36(0.11)$ \\
\hline 12 & $21.35(0.10)$ & 12 & $21.92(0.10)$ & 12 & $12.29(0.11)$ \\
\hline 13 & $23.14(0.12)$ & 13 & $23.54(0.14)$ & 13 & $13.22(0.11)$ \\
\hline 14 & $24.93(0.15)$ & 14 & $25.23(0.12)$ & 14 & $14.15(0.11)$ \\
\hline 15 & $26.64(0.22)$ & 15 & $26.89(0.17)$ & 15 & $15.12(0.11)$ \\
\hline 16 & $28.35(0.10)$ & 16 & $28.42(0.08)$ & 16 & $16.00(0.14)^{\mathrm{b}}$ \\
\hline
\end{tabular}

${ }^{\mathrm{a}} 0.00$ was rounded up from a value of -0.09 .

${ }^{b} 16.00$ was rounded down from a value of 16.10.

plotted in Figs. 2(b), 2(d), 2(f) and summarized in Table III. The SEs suggest that the random errors tend to be the highest for the mean-mean method, followed by the meansigma method. SEs are lower for the Haebara and the Stocking-Lord methods. When comparing the equating coefficients estimated under the IRT framework, the meanmean and mean-sigma methods also produced more uncertainty; the mean-mean estimated the $B$ constants with the largest SEs and the mean-sigma method estimated the $A$ constants with largest SEs.

Overall, the Stocking-Lord equating methods outperform other equating methods in terms of error reduction, with means of SE at $0.37 \%, 0.37 \%$, and $0.76 \%$ for three test pairs. These errors are smaller than $1 / 8$ of a question difference $(0.125 / 30=0.42 \%$ for BEMA and $0.125 / 16=$ $0.78 \%$ for HBEMAs). Accordingly, the score conversions for the three CIs were established using the Stocking-Lord method, which is given in Table IV. Using this conversion function, students' scores on the HBEMAs can be converted and compared directly with the scores of the full-length BEMA. Students' performances between the HBEMAs can also be directly compared.

\section{E. Equity requirement}

In this study, the equity requirement was evaluated with the assessment characteristics of different tests. This equity requirement has been partially validated by the similarity of mean item difficulty and mean score comparisons of the same population among different versions of BEMA shown in Table II. The results suggest that the three CIs have similar means of item difficulty (difference $<0.003$ logit) and total score measures (difference $<2.5 \%$ ) over three different datasets.

In addition, the equity requirement was further validated through comparing the test characteristic curves (TCCs) of the three versions of BEMA. As shown in Fig. 3, the TCCs of the HBEMA1 and HBEMA2 (dotted and dashed black lines) are shifted horizontally to the left of that of the BEMA, indicating smaller overall difficulties. In general, the three TCCs have similar nearly parallel shapes and converges at the upper ends of the ability scale. From the

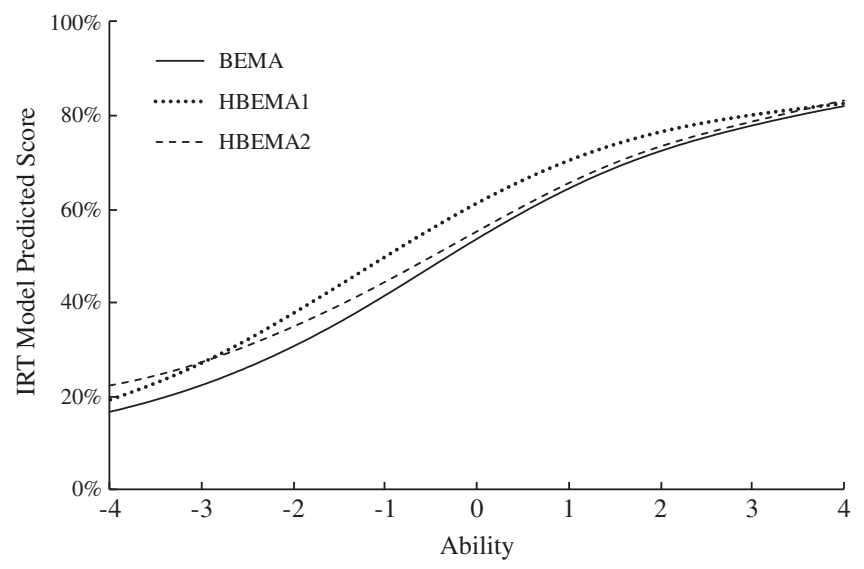

FIG. 3. Test characteristic curves (TCCs) of the two HBEMAs and the original BEMA. 
TABLE V. Root mean square deviation (RMSD) of the measured and equated scores.

\begin{tabular}{llcc}
\hline \hline & \multicolumn{3}{c}{ RMSD $(\%)$} \\
\cline { 2 - 4 } & \multicolumn{1}{c}{ Equating condition } & Measured score & Equated score \\
\hline DS1 & HBEMA1 to BEMA & 6.83 & 6.04 \\
& HBEMA2 to BEMA & 6.81 & 6.27 \\
& HBEMA1 to HBEMA2 & 10.69 & 10.50 \\
DS2 & HBEMA1 to BEMA & 7.04 & 6.06 \\
& HBEMA2 to BEMA & 6.86 & 6.27 \\
& HBEMA1 to HBEMA2 & 11.08 & 10.53 \\
\hline \hline
\end{tabular}

gap between the TCCS, the differences of model predicted scores between HBEMA1 and other two tests are larger at the center range of the ability scale. At the lower end of the ability, the two HBEMAs start to show different responding features as the TCCs cross each other. However, it is reasonable for the short CIs to perform with larger uncertainty at lower ability, since many students at this level often respond with guessing. The equating coefficients listed in Table III also provide evidence for the similarity among these TCCs. The Haebara and Stocking-Lord methods, which aim to minimize the squared difference of IRCs or the difference of TCCs of common items between two tests, both suggest near-zero adjustment on item parameters (i.e., equating coefficients $A \approx 1$ and $B \approx 0$ ).

Using the score conversion relationships listed in Table IV, students' measured scores can be converted to the scale of a target test. Then, the equity requirement can also be assessed by computing the RMSD of students' measured scores and equated scores on different CIs, which are summarized in Table V for DS1 and DS2. The results show that the errors are slightly smaller for the equated

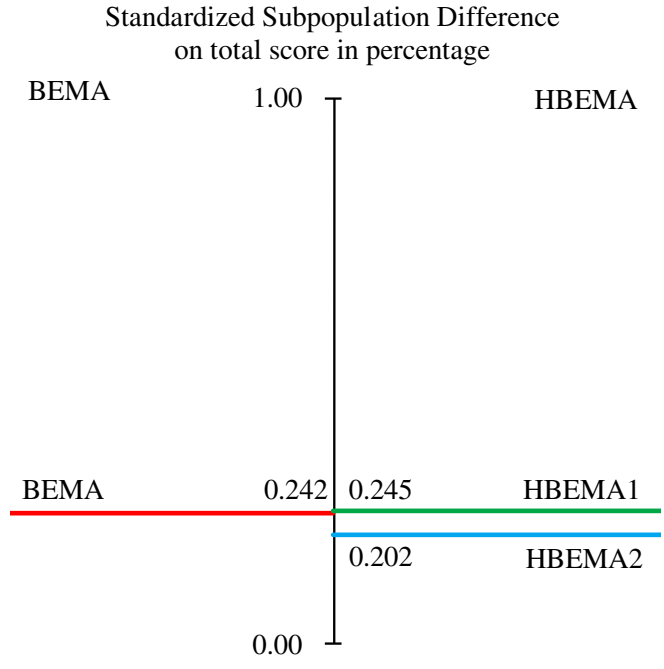

(a) scores than that for the measured scores, but are both around 2 items' difference $(2 / 30=6.67 \%$ for BEMA and $2 / 16=12.5 \%$ for HBEMAs). For different subpopulations (DS1 and DS2), the results of RMSD are robust. The values of RMSD obtained in this study are also slightly smaller than that of the previous studies $[5,6]$. Synthesizing the above results, it can be concluded that all three versions of BEMA satisfy the equity requirement.

\section{F. Population invariance requirement}

The population invariance requirement was assessed by computing the SMD between performances of different subpopulations (DS1 and DS2) on different tests. First, the standardized subpopulation differences on total scores in percentage are plotted in Fig. 4(a), which are 0.242, 0.245, and 0.202 for the BEMA, HBEMA1, and HBEMA2, respectively. As a comparison, the subpopulation differences in SAT I Math and ACT Math were found to be 0.37 and 0.34, respectively [8]. Accordingly, it can be concluded that the three versions of BEMA have similar standardized subpopulation difference percentages on total scores.

While this study primarily focused on developing short versions CIs under IRT framework, the standardized subpopulation differences on individuals' abilities estimated by 2PL models are also plotted in Fig. 4(b). Again, the results suggest that the three CIs have similar standardized subpopulation differences on estimated student abilities, which are $0.200,0.163$, and 0.171 for BEMA, HBEMA1, and HBEMA2, respectively. This similarity of SMD among the three CIs suggests that the three tests can produce nearly exchangeable scores and estimated abilities, and that the dependence of the equating method on the subpopulation is small enough to be ignored [11].

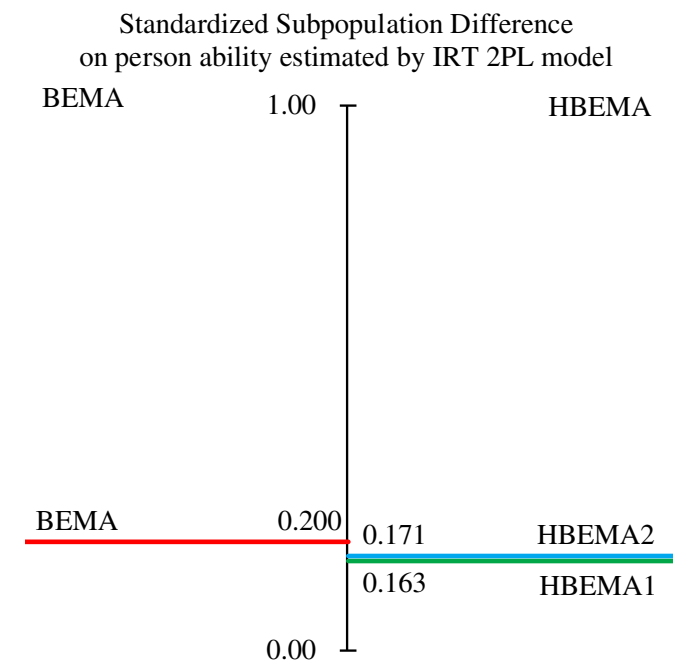

(b)

FIG. 4. Standardized mean differences between DS2 and DS3 for the three CIs on (a) total score in percentage and (b) on person ability estimated by IRT 2PL model. 
TABLE VI. Summary of linking the BEMA and CSEM using four equating methods.

\begin{tabular}{|c|c|c|c|c|c|}
\hline \multirow[b]{3}{*}{ Method } & \multicolumn{4}{|c|}{ Transformation coefficients } & \multirow{3}{*}{$\begin{array}{c}\text { Mean Conditional } \\
\text { SE of measurement } \\
\text { for total score }\end{array}$} \\
\hline & \multicolumn{2}{|l|}{$A$} & \multicolumn{2}{|l|}{$B$} & \\
\hline & Constant & SE & Constant & SE & \\
\hline mean-mean & 1.15 & 0.08 & -0.55 & 0.69 & $7.14 \%$ \\
\hline mean-sigma & 0.34 & 0.20 & 0.13 & 0.12 & $4.63 \%$ \\
\hline Haebara & 0.94 & 0.07 & 0.70 & 0.08 & $1.09 \%$ \\
\hline Stocking-Lord & 1.11 & 0.07 & 0.37 & 0.07 & $0.85 \%$ \\
\hline
\end{tabular}

Item parameters of BEMA are converted to the scale of CSEM.

\section{RESULTS OF LINKING AND COMPARING THE BEMA AND CSEM}

\section{A. Linking the scale of BEMA and CSEM}

After following the procedure for linking the BEMA and CSEM, the scale transformation coefficients are obtained and listed in Table VI. Using these scale transformation coefficients, the results for linking the BEMA and CSEM are plotted as a function of total scores on the scale of CSEM in Fig. 5. As shown in Fig. 5(a), there was an obvious difference in performance for the mean-sigma method compared to the other three methods. For the remaining three methods, the slope coefficients $A$ are near 1, which suggests approximate linear functions for score conversion between these two CIs.

The conditional standard errors produced by the four methods are also plotted as a function of total scores in Fig. 5(b). The SEs of linking suggest that the random errors tend to be the highest for the mean-mean method, followed by the mean-sigma method. SEs are lower for the Haebara method and the Stocking-Lord methods. The means of conditional SE produced by the four methods are also summarized in Table VII. Again, in terms of error reduction, the Stocking-Lord method, which has the smallest mean SE at $0.85 \%$ is suggested as the best linking method. At this level, the error is smaller than $1 / 3$ of a question

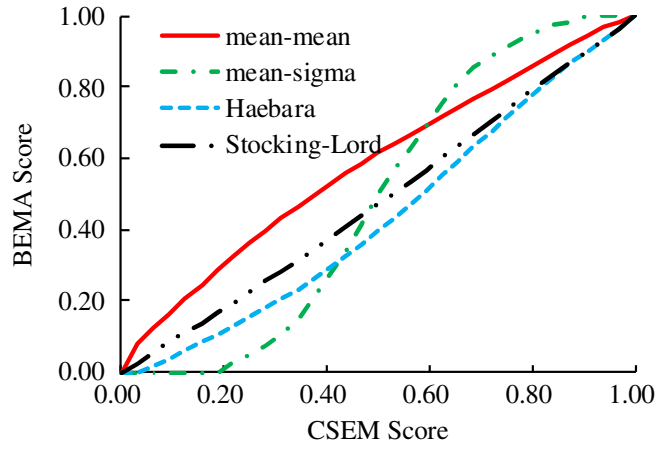

(a)
TABLE VII. Score conversion between the BEMA and CSEM using the Stocking-Lord method.

\begin{tabular}{lccc}
\hline \hline $\begin{array}{l}\text { Score of } \\
\text { CSEM }\end{array}$ & $\begin{array}{c}\text { Score as BEMA } \\
(\text { SE })\end{array}$ & $\begin{array}{c}\text { Score of } \\
\text { CSEM }\end{array}$ & $\begin{array}{c}\text { Score as BEMA } \\
(\text { SE })\end{array}$ \\
\hline 0 & $0.00(0.16)^{\mathrm{a}}$ & 17 & $15.07(0.25)$ \\
1 & $0.76(0.15)$ & 18 & $16.05(0.25)$ \\
2 & $1.66(0.16)$ & 19 & $17.04(0.25)$ \\
3 & $2.54(0.17)$ & 20 & $18.04(0.25)$ \\
4 & $3.35(0.34)$ & 21 & $19.05(0.26)$ \\
5 & $4.17(0.31)$ & 22 & $20.06(0.26)$ \\
6 & $5.02(0.29)$ & 23 & $21.08(0.26)$ \\
7 & $5.88(0.28)$ & 24 & $22.10(0.26)$ \\
8 & $6.76(0.27)$ & 25 & $23.11(0.26)$ \\
9 & $7.64(0.27)$ & 26 & $24.13(0.25)$ \\
10 & $8.54(0.26)$ & 27 & $25.12(0.24)$ \\
11 & $9.43(0.29)$ & 28 & $26.11(0.23)$ \\
12 & $10.34(0.28)$ & 29 & $27.07(0.23)$ \\
13 & $11.26(0.27)$ & 30 & $28.00(0.24)$ \\
14 & $12.19(0.26)$ & 31 & $28.90(0.28)$ \\
15 & $13.14(0.25)$ & 32 & $29.75(0.38)$ \\
16 & $14.10(0.25)$ & & \\
\hline \hline
\end{tabular}

${ }^{\mathrm{a}} 0.00$ was rounded up from a value of -0.15 .

difference $(0.33 / 30=1.11 \%$ for BEMA and $0.33 / 32=$ $1.04 \%$ for CSEM).

Using the Stocking-Lord method, the values for scale transformation coefficients $A$ and $B$ were found to be $A=1.11$ and $B=0.37$, which are slightly different from the results in Eaton et al. $\left(A^{\prime}=1.166\right.$ and $\left.B^{\prime}=0.224\right)$ [18]. This is expected as the linking relations are dependent on the interaction between population and the test $[5,6,18]$. Despite these differences, this study confirmed that the latent ability scales of CSEM and BEMA are quite similar $(A \approx 1)$. Based on the Stocking-Lord method, the score conversion between BEMA and CSEM is calculated and given in Table VII. Using this conversion function, students' scores on the CSEM can be converted and compared directly with the scores of the BEMA.

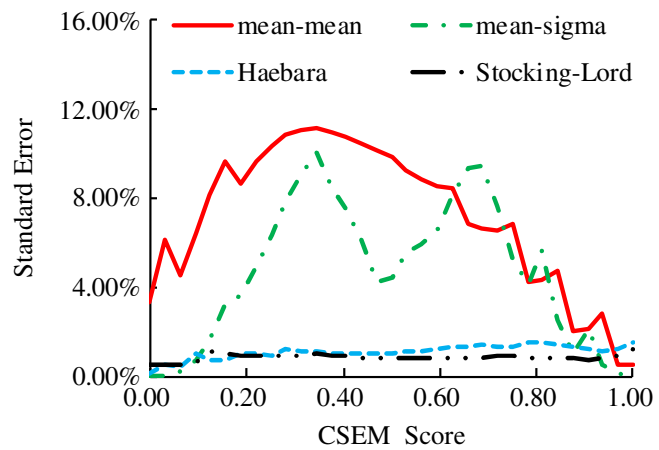

(b)

FIG. 5. Results for linking the BEMA and CSEM. (a) Linking function between BEMA and CSEM, and (b) the conditional SE for linking BEMA and CSEM. 


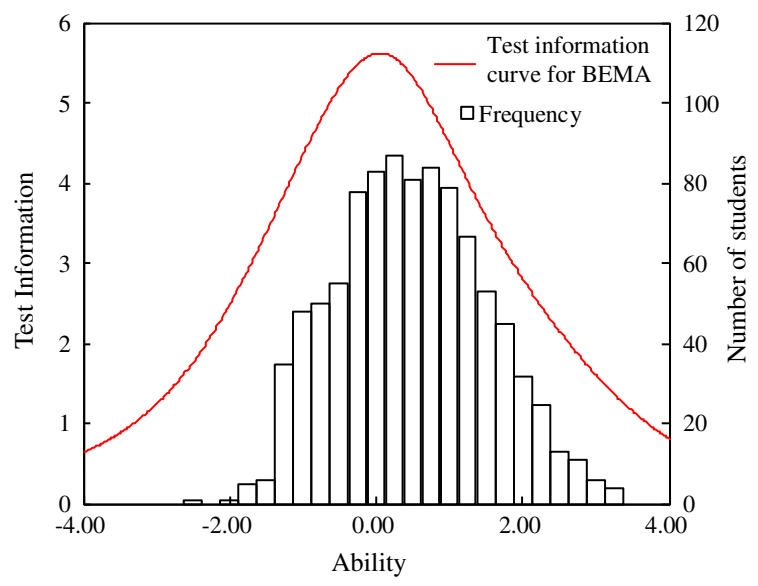

(a)

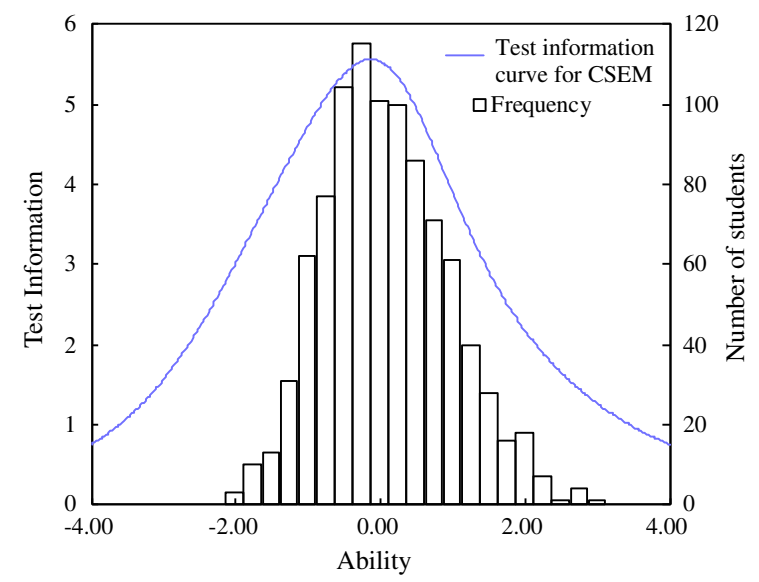

(b)

FIG. 6. Distributions of students' estimated abilities (histogram) and test information curves (line) for (a) BEMA and (b) CSEM. The histogram of students' estimated ability is overlaid with the corresponding test information curve. In Fig. 6(a), the BEMA scale has been transformed to the CSEM scale. All plots show that the bulk of the students are located in the central region of ability, which has the best feature to produce good estimates.

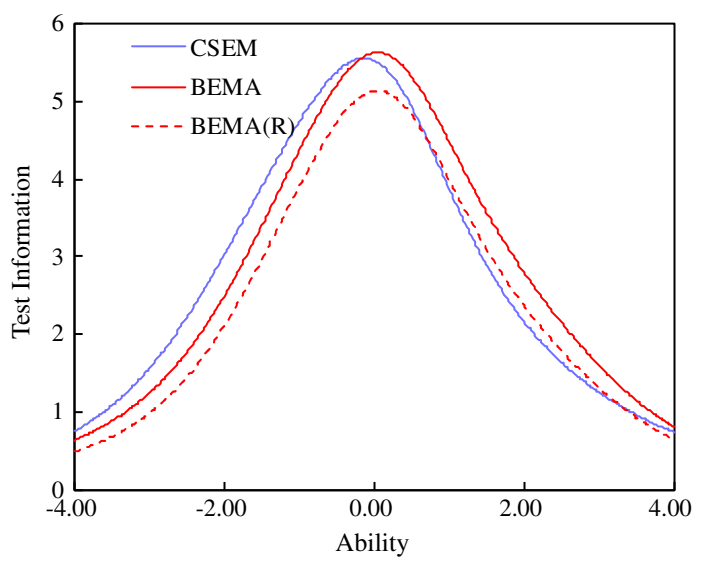

(a)

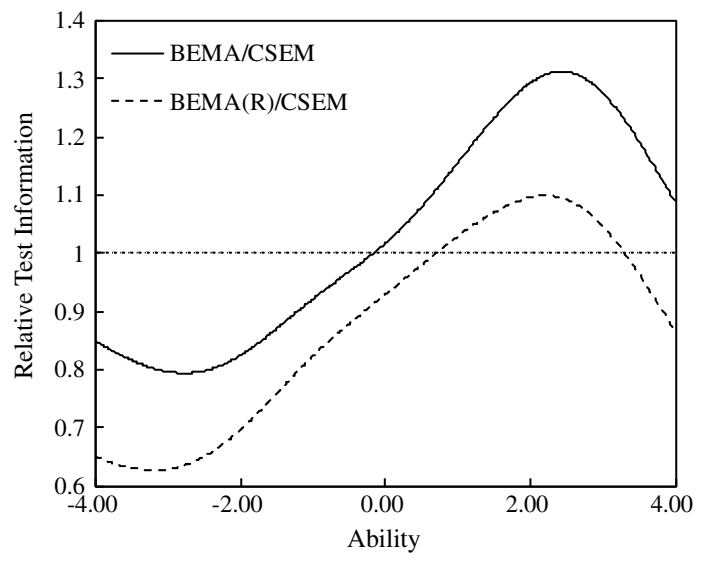

(b)

FIG. 7. Test information curves (a) and relative test information curves (b). The relative test information can be obtained by dividing the test information of the BEMA by that of the CSEM at a given ability value.

\section{B. Comparing the scale of BEMA and CSEM}

To visualize how well the BEMA and CSEM data can estimate student ability over the whole range of the ability scale, the TIFs are plotted on top of the histograms of students' estimated abilities distributed across the CSEM scale. As shown in Figs. 6(a) and 6(b), the curves of BEMA's and CSEM's TIF adequately cover the majority of the students' latent abilities for the population (DS1-3) analyzed in this study.

To directly compare the scale of BEMA and CSEM, the TIFs and the relative values of TIF were plotted in Figs. 7(a) and 7(b). As shown in Fig. 7(a), after scale transformation, the BEMA has almost identical information compared to the CSEM along the entire latent ability space (see the red line and the blue line). The similarity of TIFs is also supported by the relative value of test information functions plotted in Fig. 7(b) (see the black line), which lay between 0.80 and 1.31 with a mean of 1.03 .

This result is different from those found in Eaton et al. [18], which suggested that CSEM is better than BEMA in estimating student abilities for a range from $\theta=-2$ to $\theta=3$. The result in this study indicated that the two CIs have almost identical information after scale transformation. Eaton et al. argued that the difference on the scale of the two CIs was partially due to the difference in unidimensional treatment of the CSEM and BEMA. Specifically, more questions on CSEM than on BEMA were found to fit the unidimensional model. To further prove this point, Eaton et al. removed circuit questions from BEMA and found that the majority of the 
TABLE VIII. Equated means scores on BEMA and CSEM based on students' measured scores on HBEMAs and HCSEMs. All the statistics are given in percentage scale for easy comparisons.

\begin{tabular}{lccc}
\hline \hline & Mean score $\%$ & SD & SEM \\
\hline CSEM & 48.65 & 21.30 & 0.81 \\
BEMA & 48.29 & 21.03 & 0.80 \\
\hline \hline
\end{tabular}

information was lost between the range from $\theta=0$ to $\theta=3$, which suggested that the circuit questions on BEMA were only information heavy for that range of ability but not for the whole ability scale.

However, in this study, when the circuit questions are removed, the test information is lost uniformly along the whole ability scale [see the red dashed line for BEMA(R) in Fig. 7(a)], which is consistent with previous results suggesting that all questions on the BEMA function together to measure the same construct [26].

\section{Comparing students' performances on BEMA and CSEM}

The population of Chinese high school students was given the mixed tests composed of HBEMAs and HCSEMs. The data are used to produce equated mean scores on BEMA $(48.29 \pm 21.03 \%)$ and CSEM $(48.29 \pm 21.30 \%)$ shown in Table VIII. The scores are nearly identical and paired sample $t$ tests indicated no significant difference $[t(689)=0.588, p=0.556]$. Pearson correlation analysis indicated that there is a significant positive correlation between students' scores on the two tests $(r=0.670$, $p<0.001)$.

In addition, the test equivalence is further evaluated with the paired sample equivalence test between BEMA

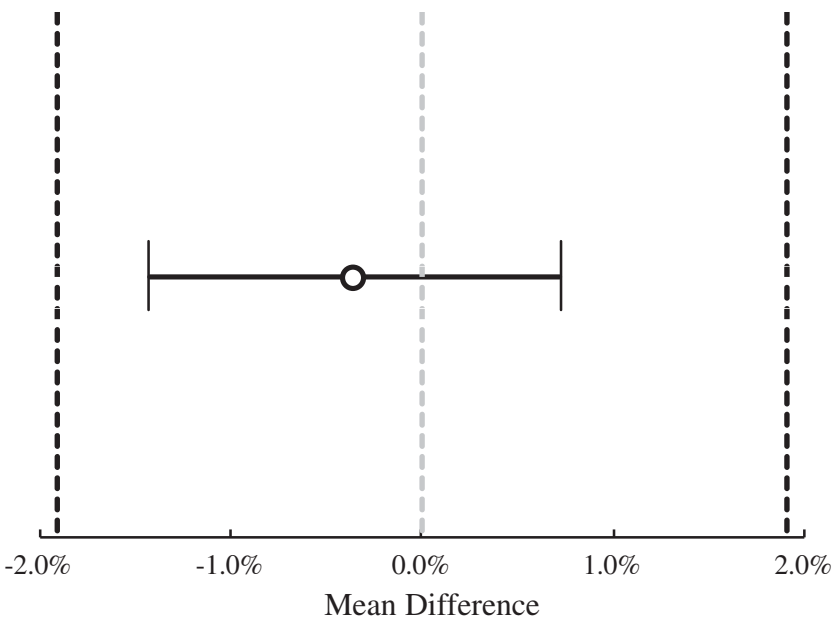

FIG. 8. Mean difference of estimated scores (shown as the circle) and $95 \%$ confidence intervals (horizontal line). The Cohen's $d_{z}$ equivalence boundaries are $\Delta_{L}=-0.11$ and $\Delta_{U}=0.11$. Corresponding score equivalence boundaries are $\Delta_{L}=-1.91 \%$ and $\Delta_{U}=1.91 \%$ (vertical dashed lines). and CSEM, which gives $t(689)=-3.47, p<0.001$, and $t(689)=2.39, p=0.009$ for the mean difference of scores between the two tests to be different from the upper and lower sides of score equivalence boundaries calculated based on the Cohen's $d_{z}$ equivalence boundaries (see Fig. 8). This confirms that students' estimated performances on the BEMA and CSEM can be considered as identical. The results of the equivalence tests are plotted in Fig. 8, which shows no overlap between the confidence interval and the equivalence boundaries.

\section{SUMMARY AND DISCUSSION}

Motived by the algorithm to develop and validate equivalent and short CIs recently proposed by Xiao et al. [5], this study developed and validated two half-length versions of BEMA (HBEMAs) under an IRT framework. Through a series of test-equating and validation analysis, the HBEMAs are confirmed to measure the same latent construct of students' understanding of electricity and magnetism to that of the original BEMA and at a similar level of reliability regarding their test design features. Considering the equating error reduction, the Stocking-Lord method was selected as the best method to create score conversions among the three versions of BEMA. It was found that the statistical characteristics of the three versions of BEMA are almost identical and robust with different subpopulations. This work expands the previous algorithm to a more strict approach in psychometric statistics (i.e., IRT framework), which advances the practice of developing short CIs.

The algorithm was also used to link two CIs (BEMA and CSEM) that target similar content in electricity and magnetism. While previous studies have tried to compare the scale of the CSEM and BEMA after a linking procedure, the common items used for linking are not identical, which is undesirable. To address this issue, in this study, items within the validated HBEMAs and HCSEMs were used as common items to link BEMA and CSEM. Again, the Stocking-Lord method was selected to create score conversions between BEMA and CSEM. After linking the two tests, it becomes possible to directly convert and compare students' performances on the two CIs. It is also found that the scale of BEMA and CSEM are almost identical along the entire latent ability scale after scale transformation. The result confirms the high similarities between these two CIs at the test level.

For a more detailed comparison, the results of this study also show differences from the work by Eaton et al. [18], which suggested that CSEM is better than BEMA in estimating student abilities for a range from $\theta=-2$ to $\theta=3$. The results of this study show that the BEMA and CSEM had almost identical information in estimating student abilities after scale transformation. Eaton et al. argued that the difference on the scale of the two CIs was partially due to the engagement of 
circuit questions in BEMA. And the circuit questions on BEMA were suggested to be only information heavy for the range from $\theta=0$ to $\theta=3$ but not for the whole ability scale. However, in this study, the information of the circuit questions was found to distribute uniformly along the whole ability scale. The difference between the two studies is not surprising since all analysis is based on the estimated item scores, and therefore, is dependent on both the population and the test design. This study tested a different set of populations and also used different linking questions (see the discussion in Sec. I and Fig. 9 in the Appendix). As a result, differences in test performance should be expected in general. The key point of this comparison is to emphasize that conclusions on general test performances cannot be drawn with a limited scope of population and are not invariant of contextual and structural variations in test design.

When the half-length versions of these CIs were used in practice, students' estimated mean scores on the scale of the two original CIs (BEMA and CSEM) were found to be within $0.5 \%$ of each other and the results of equivalence test showed that the two CIs can be used interchangeably at the $\alpha=0.05$ level with statistical power of $\beta=80 \%$. In this study, the two HBEMAs and the original BEMA were confirmed to assess the same unidimensional construct.

This endeavor for linking scores between two different CIs can also be applied in other content domains. For example, both the FCI and the FMCE are designed to assess students' understanding of introductory mechanics $[15,16]$. While shorter versions of the FCI are available [6,7], further research can be conducted to develop shorter versions of FMCE and link the FCI and FMCE using the shorter versions of FCI and FMCE. This endeavor is valuable when it is needed to compare students' performances on different CIs targeting similar content, which often occur in practice.

\section{LIMITATIONS AND SUGGESTIONS FOR FUTURE WORK}

There are several limitations of this study. First, it should be noted that the CFI and TLI values for the full BEMA are around 0.88 . These fit statistics may indicate that the full BEMA does not satisfy well the unidimensional assumption. However, since a CFI value of 0.85 has also been considered acceptable [41], taking into consideration other fit statistics (i.e., RMSEA, SRMR, DETECT, ASSI, Ratio) as well as the scree plots of PCA, the collections of analysis jointly suggest that the BEMA satisfy the assumption on unidimensionality. As discussed earlier, the outcomes of testing data are dependent on both the population and the test; therefore, the satisfaction of the unidimensional assumption is also population dependent and needs to be evaluated for each dataset.
Second, research on population fairness of CIs is getting more attention in PER. Regarding the work on half-length CIs, the short versions of FCI developed by Han et al. [6] have been concerned with gender fairness. The second half-length FCI was identified to include more gender unfair items than the first half-length FCI [27]. In this study, the fairness of the half-length versions of BEMA was not evaluated and should be investigated in future research.

Finally, the sampling of populations is still limited in this study, although effort has been made to include a more diverse range of populations. The results of this study should not be invariantly extended to students from very different education settings; however, the framework of methods established in this study can be readily applied to another populations to produce directly usable outcomes.

\section{ACKNOWLEDGMENTS}

The research is supported in part by NSF Grant No. DUE-1712238, and by the Humanities and Social Sciences of the Ministry of Education of P. R. China under Grant No. 18YJA880096.

\section{APPENDIX: ADDITIONAL TABLES AND FIGURES}

The following includes supplemental information showing the design of HBEMAs (Table IX), the item parameters for different CIs (Table X), and item parameter comparisons of the common BEMA and CSEM items in Eaton et al. (Fig. 9) [18]. Table IX lists the question orders of the HBEMAs as well as the mapped items to the

TABLE IX. BEMA to HBEMA mapping. The item order for each HBEMA is shown below, with mapped item numbers of the full-length BEMA. Items marked with asterisks (*) are common "anchoring" items that appear on both versions of HBEMAs at approximately the same locations in the respective tests.

\begin{tabular}{lcc}
\hline \hline $\begin{array}{l}\text { Item } \\
\text { number }\end{array}$ & $\begin{array}{c}\text { HBEMA1 BEMA } \\
\text { mapping }\end{array}$ & $\begin{array}{c}\text { HBEMA2 BEMA } \\
\text { mapping }\end{array}$ \\
\hline 1 & 3 & 2 \\
2 & 5 & 4 \\
3 & $6^{*}$ & $6^{*}$ \\
4 & 7 & 9 \\
5 & 8 & 12 \\
6 & 11 & $13^{*}$ \\
7 & $13^{*}$ & 14 \\
8 & 15 & $16^{*}$ \\
9 & $16^{*}$ & $17^{*}$ \\
10 & $17^{*}$ & 18 \\
11 & 18 & 19 \\
12 & 20 & $22^{*}$ \\
13 & $22^{*}$ & $24^{*}$ \\
14 & 23 & 25 \\
15 & $24^{*}$ & 26 \\
16 & 30 & 29 \\
\hline \hline
\end{tabular}


TABLE X. Item parameters for BEMA, HBEMA1, HBEMA2, and CSEM obtained in this study. The item numbers are consistent with the order of the original BEMA $(N=30)$ or $\operatorname{CSEM}(N=32)$.

\begin{tabular}{|c|c|c|c|c|c|c|c|c|}
\hline \multirow[b]{2}{*}{ Item } & \multicolumn{2}{|c|}{ BEMA } & \multicolumn{2}{|c|}{ HBEMA1 } & \multicolumn{2}{|c|}{ HBMEA2 } & \multicolumn{2}{|c|}{ CSEM } \\
\hline & $a$ & $b$ & $a$ & $b$ & $a$ & $b$ & $a$ & $b$ \\
\hline 1 & 0.569 & 3.975 & & & & & 0.579 & -2.040 \\
\hline 2 & 0.412 & 0.413 & & & 0.349 & 0.481 & 0.623 & -0.314 \\
\hline 3 & 0.449 & -0.041 & 0.368 & -0.052 & & & 1.291 & -1.580 \\
\hline 4 & 1.385 & 1.146 & & & 1.325 & 1.168 & 1.643 & -0.195 \\
\hline 5 & 1.586 & 0.233 & 1.654 & 0.222 & & & 1.395 & -0.118 \\
\hline 6 & 1.466 & 0.371 & 1.418 & 0.372 & 1.507 & 0.357 & 1.148 & -1.523 \\
\hline 7 & 0.704 & 0.459 & 0.715 & 0.450 & & & 1.892 & 0.243 \\
\hline 8 & 0.867 & 1.578 & 0.903 & 1.529 & & & 1.162 & -0.925 \\
\hline 9 & 0.055 & -22.941 & & & 0.079 & -16.169 & 1.220 & -0.973 \\
\hline 10 & 0.709 & -0.471 & & & & & 1.096 & 0.170 \\
\hline 11 & 0.358 & -4.788 & 0.333 & -5.137 & & & 0.895 & 0.884 \\
\hline 12 & 1.105 & -1.140 & & & 1.029 & -1.198 & 0.825 & -1.765 \\
\hline 13 & 0.924 & 1.526 & 0.991 & 1.450 & 0.946 & 1.497 & 0.420 & -1.635 \\
\hline 14 & 0.750 & -0.022 & & & 0.774 & -0.024 & 0.215 & 6.130 \\
\hline 15 & 1.487 & 1.097 & 1.458 & 1.107 & & & 0.817 & 0.348 \\
\hline 16 & 1.390 & -1.295 & 1.339 & -1.326 & 1.440 & -1.267 & 0.524 & 0.888 \\
\hline 17 & 0.127 & -11.957 & 0.120 & -12.594 & 0.151 & -10.013 & 0.878 & 0.267 \\
\hline 18 & 0.643 & 1.354 & 0.621 & 1.393 & 0.663 & 1.318 & 0.443 & -1.339 \\
\hline 19 & 0.746 & 2.007 & & & 0.742 & 2.014 & 0.870 & -0.002 \\
\hline 20 & 1.442 & 0.433 & 1.397 & 0.434 & & & 0.432 & 2.163 \\
\hline 21 & 1.474 & 1.340 & & & & & 0.437 & 2.971 \\
\hline 22 & 1.956 & 0.135 & 1.944 & 0.127 & 1.688 & 0.131 & 0.373 & 1.364 \\
\hline 23 & 1.145 & -0.163 & 1.116 & -0.172 & & & 1.025 & -0.437 \\
\hline 24 & 0.922 & 0.864 & 0.886 & 0.888 & 0.944 & 0.845 & 0.908 & 1.488 \\
\hline 25 & 0.686 & -0.603 & & & 0.672 & -0.616 & 1.024 & 0.364 \\
\hline 26 & 1.380 & -0.256 & & & 1.503 & -0.249 & 1.147 & -0.838 \\
\hline 27 & 0.701 & -1.524 & & & & & 0.369 & 2.107 \\
\hline 28 & 1.319 & -1.816 & & & & & 0.320 & 0.214 \\
\hline 29 & 1.626 & 0.131 & & & 1.684 & 0.120 & 0.428 & 2.672 \\
\hline 30 & 0.559 & -0.957 & 0.547 & -0.977 & & & 0.542 & -0.084 \\
\hline 31 & & & & & & & 0.857 & 2.393 \\
\hline 32 & & & & & & & 0.186 & 4.176 \\
\hline
\end{tabular}

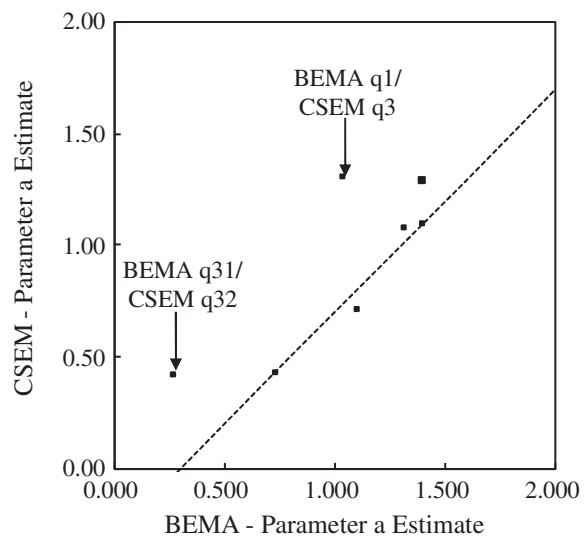

(a)

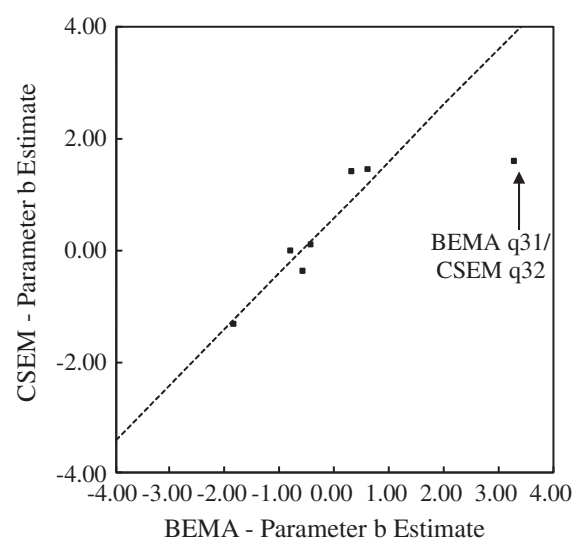

(b)

FIG. 9. Plots of parameters for the common items estimated from BEMA and CSEM. When the two estimates produce parameters based on similar latent constructs, these parameters should be in the vicinity of a straight line [4]. Therefore, these plots can be used to look for outliers, which are labeled on the diagrams. In these plots, two reference lines (dashed) are added for direct comparison. In Fig. 9(a) two item pairs (BEMA q1/CSEM q3 and BEMA q31/CSEM q32) appear to be outliers for the $a$-parameter estimate. In Fig. 9(b), the item pair BEMA q31/CSEM q32 also appears to be an outlier for the $b$-parameter estimate. Because these item pairs appear to function differently in the two tests, it is recommended to remove them from the list of common anchoring items. 
The two similar questions (BEMA-q1 and CSEM-q3) used as common anchoring questions in the study by Eaton et al.[11]. The base scenario of the two questions are identical but the choices are quite different.

Two small objects each with a net charge of $+Q$ exert a force of magnitude $F$ on each other:
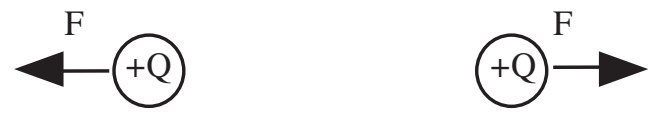

We replace one of the objects with another whose net charge is $+4 \mathrm{Q}$ :
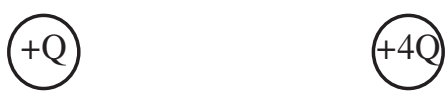

BEMA-q1: The original magnitude of the force on the $+\mathrm{Q}$ charge was F; what is the magnitude of the force on the $+\mathrm{Q}$ charge now?
(a) $4 \mathrm{~F}$
(b) $5 \mathrm{~F} / 2$
(c) $3 \mathrm{~F}$
(d) $2 \mathrm{~F}$
(e) $\mathrm{F}$
(f) $\mathrm{F} / 4$
(g) None of the above

CSEM-q3: The original magnitude of the force on the +Q charge was F; what is the magnitude of the force on the $+\mathrm{Q}$ now?
(a) $16 \mathrm{~F}$
(b) $4 \mathrm{~F}$
(c) $\mathrm{F}$
(d) $\mathrm{F} / 4$
(e) other

FIG. 10. BEMA-q1 and CSEM-q3. The number of choices of q1 in BEMA is more than that of q3 in CSEM, although the question scenarios are identical. The choice "16F" in CSEM-q3 is not included in BEMA-q1. Three additional choices are added to the BEMA q1 (i.e., " $5 / 2 F "$, " $3 F$ ", and " $2 F ")$.

original BEMA. Table $\mathrm{X}$ lists the item parameters for BEMA, HBEMA1, HBEMA2, and CSEM obtained in this study. Figure 9 shows details of the item parameters $a$ and $b$ for the seven almost identical items in the two CIs. In Fig. 10, two identical items in the two CIs are used to illustrate the potential difference.
[1] D. L. Evans, G. L. Gray, S. Krause, J. Martin, C. Midkiff, B. M. Notaros, M. Pavelich, D. Rancour, T. Reed-Rhoads, and P. Steif, Progress on Concept Inventory Assessment Tools, in 33rd Annual Frontiers in Education, 2003. FIE 2003 (IEEE, Boulder, CO, 2003), pp. T4G-1, https:// doi.org/10.1109/FIE.2003.1263392.

[2] L. Ding, R. Chabay, B. Sherwood, and R. Beichner, Evaluating an electricity and magnetism assessment tool: Brief electricity and magnetism assessment, Phys. Rev. ST Phys. Educ. Res. 2, 010105 (2006).
[3] D. P. Maloney, T. L. O'Kuma, C. J. Hieggelke, and A. V. Heuvelen, Surveying students' conceptual knowledge of electricity and magnetism, Am. J. Phys. 69, S12 (2001).

[4] M. J. Kolen and R. L. Brennan, Test Equating, Scaling, and Linking: Methods and Practices (Springer Science \& Business Media, New York, 2014), https://doi.org/ 10.1007/978-1-4939-0317-7.

[5] Y. Xiao, K. Koenig, J. Han, Q. Liu, J. Xiong, and L. Bao, Test equity in developing short version conceptual inventories: A case study on the Conceptual Survey of 
Electricity and Magnetism, Phys. Rev. Phys. Educ. Res. 15, 010122 (2019).

[6] J. Han, L. Bao, L. Chen, T. Cai, Y. Pi, S. Zhou, Y. Tu, and K. Koenig, Dividing the Force Concept Inventory into two equivalent half-length tests, Phys. Rev. ST Phys. Educ. Res. 11, 010112 (2015).

[7] J. Han, K. Koenig, L. Cui, J. Fritchman, D. Li, W. Sun, Z. $\mathrm{Fu}$, and L. Bao, Experimental validation of the half-length Force Concept Inventory, Phys. Rev. Phys. Educ. Res. 12, 020122 (2016).

[8] N. J. Dorans, Distinctions among Classes of Linkages. Research Notes. RN-11 (College Entrance Examination Board, New York, NY, 2000).

[9] P. W. Holland and N. J. Dorans, Linking and equating, in Educational Measurement, edited by R. Brennan, 4th ed. (Praeger, Westport, CT, 2006), pp. 187-220.

[10] Y. Tong and M. J. Kolen, Assessing equating results on different equating criteria, Appl. Psychol. Meas. 29, 418 (2005).

[11] N. J. Dorans, T. P. Moses, and D. R. Eignor, Principles and practices of test score equating, ETS Res. Rep. Ser. 2010, 1 (2010).

[12] M. Battauz, IRT test equating in complex linkage plans, Psychometrika 78, 464 (2013).

[13] B. G. Brossman and W.-C. Lee, Observed score and true score equating procedures for multidimensional item response theory, Appl. Psychol. Meas. 37, 460 (2013).

[14] R. K. Thornton, D. Kuhl, K. Cummings, and J. Marx, Comparing the force and motion conceptual evaluation and the force concept inventory, Phys. Rev. ST Phys. Educ. Res. 5, 010105 (2009).

[15] R. K. Thornton and D. R. Sokoloff, Assessing student learning of Newton's laws: The force and motion conceptual evaluation and the evaluation of active learning laboratory and lecture curricula, Am. J. Phys. 66, 338 (1998).

[16] D. Hestenes, M. Wells, and G. Swackhamer, Force Concept Inventory, Phys. Teach. 30, 141 (1992).

[17] S. J. Pollock, Comparing student learning with multiple research-based conceptual surveys: CSEM and BEMA., AIP Conf. Proc. 1064, 171 (2008).

[18] P. Eaton, K. Johnson, B. Frank, and S. Willoughby, Classical test theory and item response theory comparison of the brief electricity and magnetism assessment and the Conceptual Survey of Electricity and Magnetism, Phys. Rev. Phys. Educ. Res. 15, 010102 (2019).

[19] W. J. van der Linden and R. K. Hambleton, Handbook of Modern Item Response Theory (Springer, New York, 1997).

[20] X. Liu, Using and Developing Measurement Instruments in Science Education: A Rasch Modeling Approach (Infromation Age Publishing, Charlotte, NC, 2010).

[21] J. Wang and L. Bao, Analyzing force concept inventory with item response theory, Am. J. Phys. 78, 1064 (2010).

[22] V. Mešić, K. Neumann, I. Aviani, E. Hasović, W. J. Boone, N. Erceg, V. Grubelnik, A. Sušac, D. S. Glamočić, M. Karuza, A. Vidak, A. Alihodžić, and R. Repnik, Measuring students' conceptual understanding of wave optics: A Rasch modeling approach, Phys. Rev. Phys. Educ. Res. 15, 010115 (2019).

[23] U. Scotti di Uccio, A. Colantonio, S. Galano, I. Marzoli, F. Trani, and I. Testa, Design and validation of a two-tier questionnaire on basic aspects in quantum mechanics, Phys. Rev. Phys. Educ. Res. 15, 010137 (2019).

[24] Y. Xiao, J. Han, K. Koenig, J. Xiong, and L. Bao, Multilevel Rasch modeling of two-tier multiple choice test: A case study using Lawson's classroom test of scientific reasoning, Phys. Rev. Phys. Educ. Res. 14, 020104 (2018).

[25] G. W. Fulmer, H.-E. Chu, D. F. Treagust, and K. Neumann, Is it harder to know or to reason? Analyzing two-tier science assessment items using the Rasch measurement model, Asia-Pac. Sci. Educ. 1, 1 (2015).

[26] L. Ding, Seeking missing pieces in science concept assessments: Reevaluating the Brief Electricity and Magnetism Assessment through Rasch analysis, Phys. Rev. ST Phys. Educ. Res. 10, 010105 (2014).

[27] A. Traxler, R. Henderson, J. Stewart, G. Stewart, A. Papak, and R. Lindell, Gender fairness within the Force Concept Inventory, Phys. Rev. Phys. Educ. Res. 14, 010103 (2018).

[28] J. Stewart, C. Zabriskie, S. DeVore, and G. Stewart, Multidimensional item response theory and the Force Concept Inventory, Phys. Rev. Phys. Educ. Res. 14, 010137 (2018).

[29] C. Zabriskie and J. Stewart, Multidimensional item response theory and the Conceptual Survey of Electricity and Magnetism, Phys. Rev. Phys. Educ. Res. 15, 020107 (2019).

[30] T. F. Scottand D. Schumayer, Students' proficiency scores within multitrait item response theory, Phys. Rev. ST Phys. Educ. Res. 11, 020134 (2015).

[31] T. F. Scott, D. Schumayer, and A. R. Gray, Exploratory factor analysis of a Force Concept Inventory data set, Phys. Rev. ST Phys. Educ. Res. 8, 020105 (2012).

[32] L. Bao, T. Cai, K. Koenig, K. Fang, J. Han, J. Wang, Q. Liu, L. Ding, L. Cui, and Y. Luo, Learning and scientific reasoning, Science 323, 586 (2009).

[33] See Supplemental Material at http://link.aps.org/ supplemental/10.1103/PhysRevPhysEducRes.15.020149 for the process and results of unidimensionality tests.

[34] F. B. Baker and S.-H. Kim, The Test Characteristic Curve, in Basics Item Response Theory Using $R$ (Springer, Cham, 2017), pp. 55-67.

[35] N. J. Dorans and P. W. Holland, Population invariance and the equatability of tests: Basic theory and the linear case, J. Educ. Measure. 37, 281 (2000).

[36] G. A. Morris, N. Harshman, L. Branum-Martin, E. Mazur, T. Mzoughi, and S. D. Baker, An item response curves analysis of the Force Concept Inventory, Am. J. Phys. 80, 825 (2012).

[37] F. M. Lord and M. S. Wingersky, Comparison of IRT truescore and equipercentile observed-score "equatings", Appl. Psychol. Meas. 8, 453 (1984).

[38] F. B. Baker and S.-H. Kim, The Basics of Item Response Theory Using $R$ (Springer International Publishing, New York, NY, 2017), pp. 89-104.

[39] D. Lakens, Equivalence tests: A practical primer for t tests, correlations, and meta-analyses, Soc. Psychol. Personal. Sci. 8, 355 (2017).

[40] M. A. Seaman and R. C. Serlin, Equivalence confidence intervals for two-group comparisons of means, Psychol. Methods 3, 403 (1998).

[41] K. A. Bollen, Structural Equations with Latent Variables (Wiley, New York, NY, 1989). 